Int. J. Speleol. 10 (1978), pp. 279 - 290

\title{
Niveaux de base actuels dans les principaux canyons du Languedoc oriental et des Plans de provence
}

par

\author{
G. FABRE* et J. NICOD*
}

Present Karstic base level in the main canyons of oriental Languedoc and Plans of Provence.

\section{SUMMARY}

In a karstic country cut by a canyon in which runs an allogenic river, there are several base levels (karstic, fluvial...); chiefly if the limestones develop greatly under the bottom of the gorge. The difficulty of characterizing them appears in all the definitions given. From the example of the three most important canyons of the Eastern Bas Languedoc (South-East of France) and with the different geological controls (s.l.) one ascertain that at present:

a) When an allogenic and surface river is perennal without important loss, there is a total connexion between fluvial level and karstic base level.

b) When an allogenic and temporary surface river doesn't flow and has losses, there is no conncxion between the major fluvial and base level and the karstic base level.

Les canyons constituent une forme majeure du karst, et pourtant méconnue. Trop souvent ils sont assimilés aux gorges qui entaillent n'importe quelles roches. Pourtant les grottes - recoupements d'anciens réseaux - qui débouchent sur leurs parois nous invitent à envisager leur creusement en fonction de l'évolution du karst. Il est indispensable de rappeler ici deux principes fondamentaux.

1 - l'écoulement au fond des canyons est pérenne, saisonnier ou épisodique en fonction des conditions climatiques, de l'étendue du bassin versant allogène à l'amont du karst, mais aussi de la part des infiltrations et des résurgences, donc du fonctionnement de l'aquifère karstique.

2 - Selon que l'écoulement est pérenne, saisonnier ou épisodique, le profil en long est très différent. Dans le premier cas, sauf de rares exceptions (haute montagne, néotectonique le profil en long du fond du canyon est régulier,

* ERA 282 du Centre National de la Recherche Scientifique (C . N.R.S.) "Ivolution Karstique dans les domaines méditerranéen et alpin".

Institut de Ciégraphie, 29 Avenue Robert Schuman - 13621 AIX-en-PRo)VI:NCI: I RAN( I:. 
comme celui des rivières et des fleuves non karstiques auxquels il se rattache. Comme c'est le cas du Verdon dans le 'Grand Canyon ou de l'Ardèche en aval de Vallon Pont d'Arc, dans les autres cas il présente des irrégularités qui font jusqu'à des chutes (gradins de confluence karstique, Nicod, 1967, p. 507). Il y a donc des cas où le profil des cours d'eau karstiques est déconnecté par rapport au profil général du réseau hydrographique. C'est poser le problème du niveau de base dans les karsts, qui peut être éclairé à la lumière des travaux hydrologiques récents.

\section{I - CONCEPTION DU NIVEAU DE BASE}

Il s'agit d'un concept très discuté, en milieu karstique. Dans l'écoulement subaérien beaucoup d'auteurs on étudié le profil d'équilibre des cours d'eau, autre concept qui se rattache à celui du niveau de base. Que devient-il dans les canyons karstiques?

De nombreux auteurs ont d'ailleurs souligné les aspects complexes, confus et surtout ambigüs de cette notion générale «d'équilibre» (1) qui ne peut vraiment être abordée qu'à partir d'exemples régionaux, comme l'avait affirmé $\mathrm{J}$. Cvijić dans son dernier travail posthume (1960). Ceux-ci ont démontré la multiplicité des types de niveaux de base dans l'espace comme dans le temps. Sur ce point, P. Fenelon (1975) a bien souligné la distinction qu'il convient d'opérer entre les niveaux de base géomorphologiques et karstiques.

I - I.e niveau de base géomorphologique général est celui de la mer (ou des lacs) auquel se raccordent les profils du réseau hydrographique: c'est le niveau de base fluviatile. Il intervient aussi dans le karst.

- de la même manière, dans l'écoulement des cours d'eau subaériens des canyons, sous certaines conditions, cf. infra.

- directement dans l'écoulement souterrain dans les karsts littoraux

- indirectement dans la position altimétrique de nombreuses sources karstiques (émergences permanentes de fond de vallée, exemple très classique de la Sorgue pérenne sortant de la Fontaine de Vaucluse).

2 - Ie niveau de base structural du karst est constitué par le toit de l'imperméable sous les calcaires karstifiables. Il règle la position des exutoires dans les karst perchés (Castany 1963, p. 975 et Nicod 1969, p. 310) et peut constituer, en profondeur, une limite au développement du karst noyé profond.

- I.e niveau de base hydrodynamique du karst, ou niveau de base majeur du harst (Fabre 1977) est plus délicat à définir.

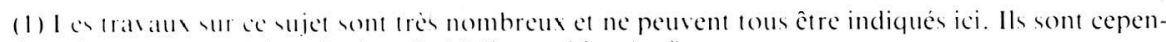
damt largement mentionne dams k hibliographic "in fine». 
Dans un système de cours d'eau subaériens, les profils des affluents se raccordent à celui du cours d'eau principal.

Dans un système karstique à prédominance de circulation souterraine, existe selon Gèze (1973) quatre zones hydromorphologiques:

- Zone d'absorption superficielle limitée à la base par une sous-zone de subsurface renfermant ou non un aquifère épikarstique temporaire.

- Zone de transfert vertical très transmissive.

- Zone de transfert sub-horizontal à la hauteur des exsurgences.

- Zone noyée d'imbibition générale occupant un réseau de fissures et de conduits plus ou moins pénétrables, hiérarchisés et reliés entre eux.

C'est le niveau de la zone de transfert sub-horizontal qui règle les conditions d'écoulement dans le fond des canyons, comme nous allons le voir à la lumière des exemples languedociens et provençaux étudiés; à ce titre il constitue le niveau de base majeur du karst.

\section{II - L'EXEMPLE DES TROIS CANYONS DU LANGUEDOC ORIENTAL: ARDĖCHE, CÈZE et GARD}

Leur intérêt est de présenter dans des conditions de similitude géographique (vaste bassin amont allogène dans la montagne cévenole, encaissement dans de profonds canyons dans leur cours moyen, variations considérables du régime), de grandes différences d'écoulement du fond de leurs canyons.

1 - Données générales sur les trois canyons (fig. 1) (2)

Dans leur cours moyen, ces trois affluents du Rhône ont creusé de profonds canyons dans les calcaires compacts, très purs, plissés et très fracturés du faciès urgonien du Barrémien supérieur (fig.2). Puissants d'au moins $250 \mathrm{~m}$, ils se développent largement sous le talweg où ils affleurènt localement en place au droit de seuils sous les formes de dalles et de chicots déchiquetés (Ex. Charlemagne et Pas du Mousse dans l'Ardéche, pont Saint-Nicolas dans le Gardon). Aucun forage n'a permis d'établir précisément la profondeur du mur de ce faciès ni la nature exacte des formations sous-jacentes à son contact. II s'agit en toute vraisemblance des assises calcaréo-marneuses du Barrémien moyen inférieur. Dans les lits actuels, par contre, des sondages ont montré que les alluvions cévenoles à base de quartz $(70 \%)$ pouvaient dépasser $10 \mathrm{~m}$ d'epaisseur. Toutefois, au long du talweg, elles n'atteignent pas dans l'esemble $5 \mathrm{~m}$.

Au niveau des gorges, le régime de ces trois rivières allogènes est différent, surtout à l'étiage. Ainsi, au cours de nombreux cycles annuels on a observé que (2):

(2) - Cartographic, bibliographic et renseignement plus détailles sont donneis dans (i.lahre(1977). 
Fig. 1-Situation des trois principaux canyons urgoniens du Languedoc oriental.

- De Vallon Pont d'Arc à Saint-Martin d'Ardèche, l'Ardèche reste toujours épigée. S'il y a des pertes, il semble bien qu'elles soient faibles.

- De Tharaux au pont de Saint-André de Roquepertuis, la Cèze est épigée, mais elle subit des prélévement hypogés qui peuvent conduire à un tarissement total de Rochegude à Tharaux et dans certaines portions aval de son cours. L.e Gardon quant à lui tarit régulièrement et complètement de Boucoiran à Moussac en amont des gorges, puis de Dions jusqu'en aval des Frégiéres sur quelque $15 \mathrm{~km}$, où il réapparaît à la faveur d'un système échelonné d'exsurgences pérennes (résurgences - émergences).

Sur le plan spatial de l'organisation actuelle des circulations karstiques, on a pu mettre correctement en évidence l'existence générale des quatre zones hydrogéomorphologiques décrites par Gèze (1973) (cf. supra)

2 - Existence de formes d'érosion et de corrosion actives des trois canyons languedociens.

Elles se trouvent au niveau de la zone de transfert sub-horizontale, et on a pu en étudier divers exemples:

- dans l'Ardèche aux sources de fond situées $200 \mathrm{~m}$ en aval des évents de Foussoubie;

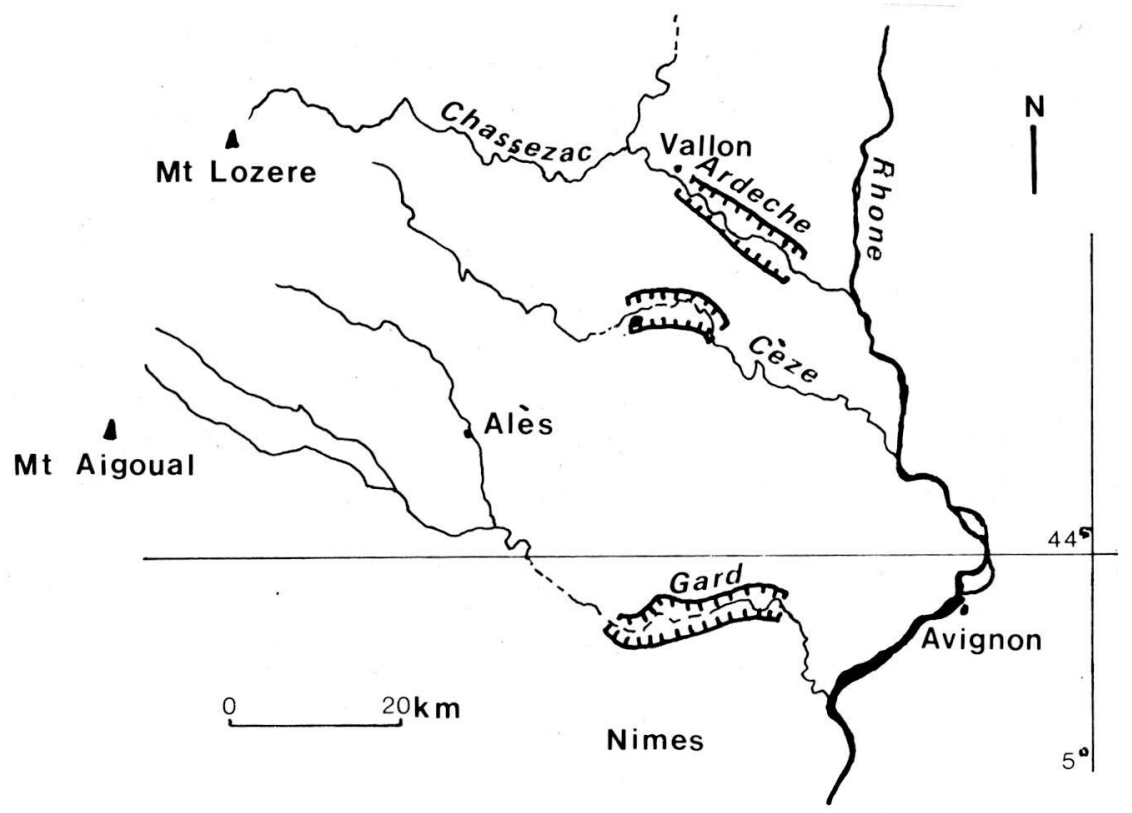




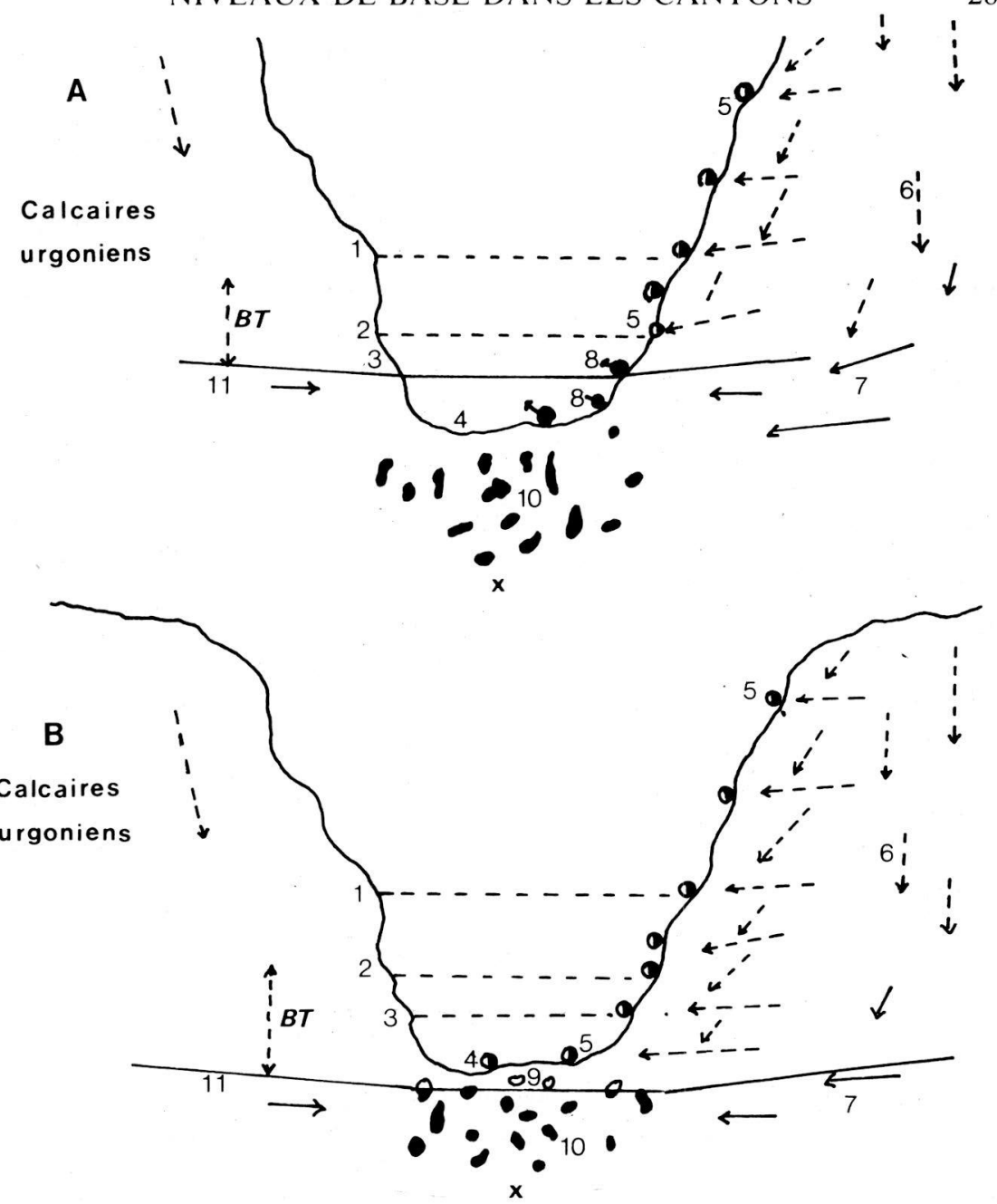

Fig. 2 - REPRESENTATION SCHEMATIQUE DES NIVEAUX DE BASE ACTUELS DANS LES TROIS PRINCIPAUX CANYONS DU LANGUEDOC ORIENTAL (plus basse seaux - tarissement). A: cas d'une rivière allogène pérenne (Ardèche, Cèze en aval de Tharaux, Gard en aval des Frégières). B: cas d'une rivière allogène temporaire tarie (Gard en amont des Frégières, Cèze à la Baume Salène, en amont de Tharaux). Légende commune. $-1=$ Niveau maximum du lit majeur et sommet du niveau de base fluviatile (temporaires) - 2 = Niveau habituel de la rivière et niveau de base fluviatile habituel (temporaires). - 3 = Niveau minimum du lit d'étiage (pérenne en A, temporaire B). 4 = Talweg (roche en place) et mur du niveau de base fluviatile (pérenne en A, temporaire en B) - 5 = Sources karstiques temporaires et niveaux de base karstiques, secondaires, très localisés, peu actifs et temporaires $-6=$ Ecoulements karstiques temporaires $-7=$ Ecoulements karstiques pérennes $-8=$ Sources karstiques pérennes $-9=$ Fissures et conduits karstiques secs temporairement $-10=$ Fissures et conduits karstiques noyés pérennes $-11=$ Surface piézométrique à l'étiage (BT - balancement temporaire) - Niveaux de base karstiques majeurs actuellement actifs et pérennes fonction de $11-\mathrm{X}=$ mur inconnu du niveau de base karstique majeur. NB. - Sur les deux figures, les niveaux des écoulements sont volontairement très exagérés. 
- dans la Cèze à la grotte des Fées, sous le Galinier et aux sources de Monteils et de Marnade;

- dans le Gardon sous les ponts de Dions et de Russan et aux exutoires de l'En Quissé, de la Fausse Monnaise, des Frégières, de Pâques, etc...

Des observations identiques ont été formulées dans les autres canyons du midi méditerranéen français et des Causses (A. Cavaillé, 1964, P. Dubois, 1969, J. Nicod, 1967).

De même des exsurgences et des axes de drainage majeurs ou non ont été individualisés dans la Cèze à Marnade et le Gardon aux Frégières, à la Baume Saint-Vérédème et à Pâques (Fabre,1976), ils sont bien évidemment liés aux fluctuations de la surface «piézométrique» située dans le karst et des niveaux des rivières.

\section{3 - Rapports de l'écoulement subaérien dans le canyon et de l'écoulement sou- terrain.}

La Fig. 2 présente les deux cas rencontrés. Dans le premier (A) le cours d'eau est pérenne et coule au niveau de la zone de transfert horizontal qui s'équilibre sur lui; et réciproquement.

C'est le cas de l'Ardèche et du Gard en aval des Frégières et sourtout des sources de la Baume Saint-Vérédème.

Dans le second (B) il y a disjonction entre le fond du canyon et le niveau de la zone de transfert. L'action d'érosion et de corrosion actives se situe à ce niveau, qui devient alors le niveau de base géomorphologique majeur du karst . C'est le cas du Gard en amont de Frégières et de la Cèze en amont des sources des Fades.

\section{4 - Concept du niveau de base karstique majeur}

Il s'agit d'un niveau, qui a une double définition hydrodynamique: il correspond à la partie supérieure de la zone noyée, on niveau «piézométrique» avec toutes les irrégularités que comporte celui-ci, en raison de l'anisotropie du karst. Il varie donc en fonction des conditions hydrologiques. Géomorphologique; il est commandé de l'aval par la position des plus basses sources pérennes, et ne peut descendre en dessous des plus bas conduits noyés.

En fonction de ceci et des travaux sur ces karsts, on constate que dans les conditions actuelles:

a) Lorsqu'un un cours d'eau épigé allogène est pérenne et sans perte notable, il y a correspondance globale entre le niveau de base fluviatile s.l. et le niveau de base karstique majeur matérialisé par les conduits noyés situés sous le canyon. b) Lorsqu'un cours d'eau épigé allogène temporaire ne coule pas par suite de prélèvements karstiques, il n'y a pas correspondance entre les différents niveaux de base. Le niveau de base fluviatile s.l. est temporairement actif. Quant au niveau de base karstique majeur pérenne il se place dans la zone 
noyée comprise entre le mur atteint par la corrosion et le toit des écoulements karstiques pérennes situés sous le talweg sec; c'est-à-dire principalement au niveau des plus basses et importantes sources pérennes drainant le karst.

\section{5) - Existence de niveaux de base secondaires}

Au droit des grands canyons, on peut qualifier ces niveaux actifs en permanence, de majeurs; mais il est bien évident qu'il y en a d'autres, mineurs (secondaires). Subordonnés aux premiers, ils sont plus_ou moins importants, actifs, difficiles à intégrer dans un classement bien hiérarchisé et intimement liés aux conditions hydrologiques et aux differents contrôles locaux de leur établissement. Citons:

- Le mur des aquifères épikarstiques (Ex. les Deux Anes et le Castelas dans le Gardon).

- Le toit des dalles urgoniennes légèrement marneuses supportant le piton de la Hutte (Gardon) ou celles du Serre du Roi dans la Cèze

- Certaines sources de vallées adjacentes (Ex. exsurgences de Tiourre sur la rive gauche de l'Ardèche et de Force Male dans la Cèze).

D'une façon générale dans ces vallées latérales et les canyons du midi méditerranéen français d'autres nuances apparaisent entre ces types selon que les cours d'eau sont secs en permanence sur une partie ou la totalité de leur trajet, subissent des prélèvements partiels ou complets, diffus ou très localisés, ou qu'ils coulent tout le temps, sans perte ou peu. En tout état de cause cette définition «éclatée» et modulée du niveau de base karstique découle du problème de la dynamique permanente de l'eau qui commande toute l'évolution du creusement des gorges calcaires. C'est d'ailleurs elle, ainsi que le montre la planche 2, qui explique que les niveaux de base, karstiques en particulier, et leur rapport avec l'érosion s.l. sont caractérisés par un phénomène «d'amplitude» spatio-temporel déterminé par le balancement permanent de la surface "piézométrique» surtout au droit des plus grosses sources pérennes.

\section{III - L'EXEMPLE DES CANYONS DES PLANS DE PROVENCE (VER- DON et ARTUBY).-}

Bien qu'il s'agisse, comme dans les cas précédents de cours d'eau allogènes traversant la masse calcaire en canyons, les conditions hydrogéologiques sont radicalement différentes, les écoulements souterrains s'effectuant non sous les canyons, mais directement en court-circuit, vers la résurgence de Fontaine l'vêque.

\section{I - Description du système (Fig 3)}

Sur une quarantine de kilomètres, le Verdon, puissant cours d'eau allogène traverse la masse calcaire jurassique des Plans de Canjuers, épaisse d'un millier de mètres, et affectée de divers accidents tectoniques (plis et failles). Il est pé- 


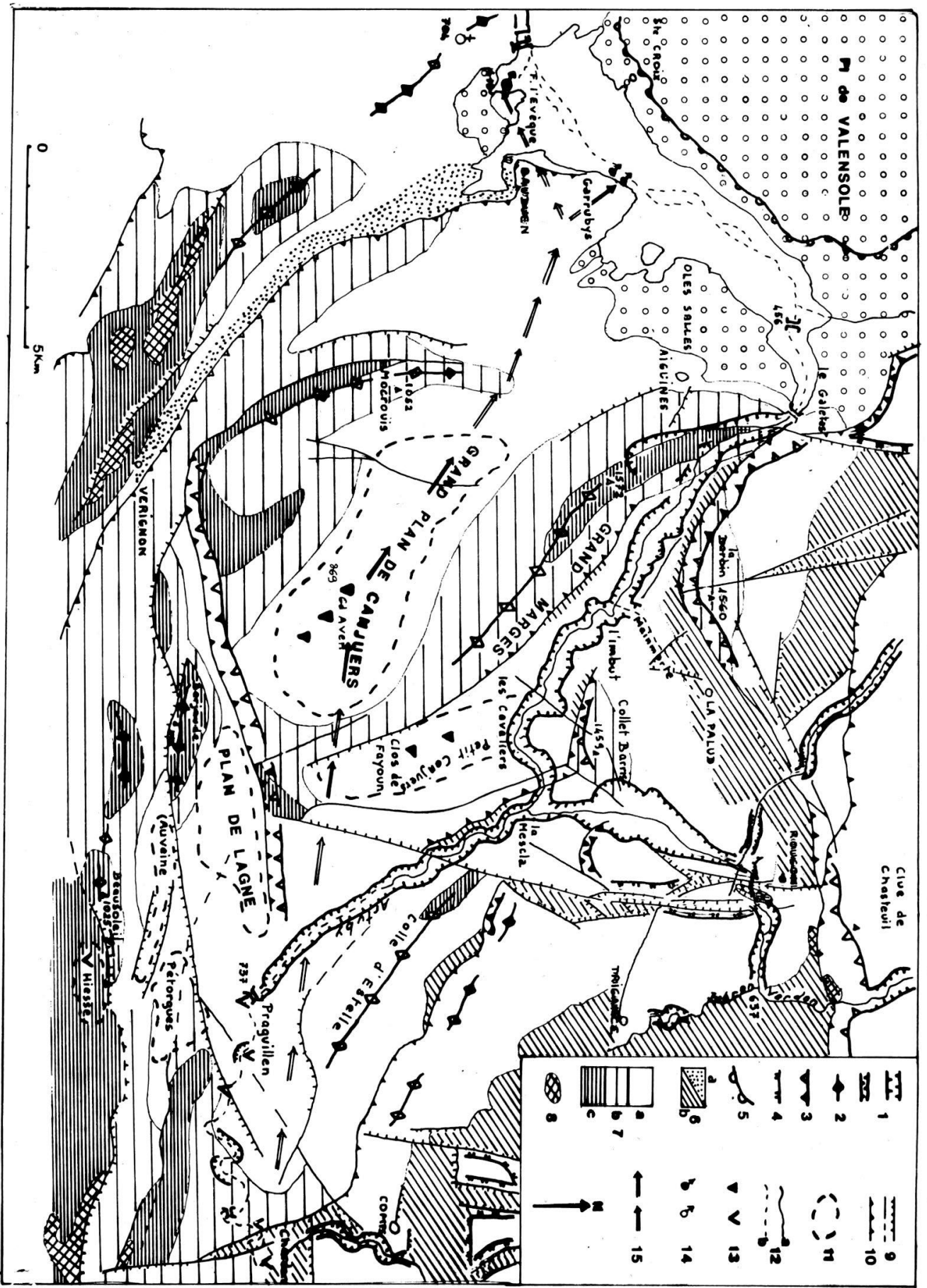


renne: les pertes au fond n'ont qu'une inportante minime (Nicod 1967, p. 489) et à l'exception du resserrement de l'Imbut, son profil est régulier.

Tout autre est le cas de l'Artuby. Son canyons entre le Pont de Praguillen et le confluent, «la Mescla», est généralement à sec. Le lit relativement régulier est encombré de cailloux abandonnés en amont de la faille du Pas de la Chapelle devient très irrégulier jusqu'au confluent, suite de sauts (avec la baume du Chaos) et de marmites de Géant.

A l'étiage, l'Artuby est absorbé en amont du karst. Il parvient jusqu'au pont de Praguillen pour des débits de l'ordre de $500 \mathrm{l} / \mathrm{s}$, et à la Mescla pour $850 \mathrm{l} / \mathrm{s}$. On peut estimer (Nicod 1967, p. 489) que l'absorption de l'Artuby correspond a un module annuel de $5 \mathrm{~m}^{3} / \mathrm{s}$ dont les $9 / 10$ alimentant Fontaine l'Evêque. Càr les eaux de l'Artuby, grossies des précipitations sur l'impluvium des Plans, parviennent directement à la résurgence principale de Fontaine l'Evêque (module $5,7 \mathrm{~m}^{3} / \mathrm{s}$ ) et aux émergences temporaires des Garrubys (aujourd'hui noyées le réservoir de Sainte-Croix). Il s'agit d'un véritable court-circuit.

\section{2 - Disjonction totale du profil subaérien et du niveau de base karstique ma- jeur en ce qui concerne l'Artuby.}

L'Artuby descend de $730 \mathrm{~m}$ au Pont de Praguillen à $563 \mathrm{~m}$ au confluent (La Mescla). Le niveau de base karstique majeur est défini par:

a - l'altitude de l'exutoire $(410 \mathrm{~m})$

b - la pente de la partie supérieure de la zone noyée, qui est nécessairement faible, en raison de l'existence d'un ou plusieurs drains privilégiés, comme celui qui aboutit à la résurgence et a été exploré en 1966 (Fig. 4).

La puissance de la série calcaire, de l'ordre du millier de mètres sous les Plans de Canjuers a permis le développement d'un système karstique profond, dont

1: Canyons

2: Mont (anticlinal)

3 : Escarpement chevauchement

4: Escarpement de faille

$5:$ Formation et rebord (Valensole)

6: Barrages synclinaux a) sable Oligocène b) marnes Crétacé

7 : Calcaires jurassiques a)base à $300 \mathrm{~m} \mathrm{~b}$ ) 300 à $600 \mathrm{~m} \mathrm{c}$ ) $+600 \mathrm{~m}$

8: Keuper imperméable

9: Failles

10 : Chevauchements

11 : Grands bassins karstiques

12 : Cours d'eau a) perenne b) temporaire

13 : Aven, perte

14 : Source principale, de trop plein

15 : Direction probable des écoulements karstiques

Fig. 3: carte du complexe géomorphologique et hydrologique des Plans de Provence et du Grand Canyon du Verdon 


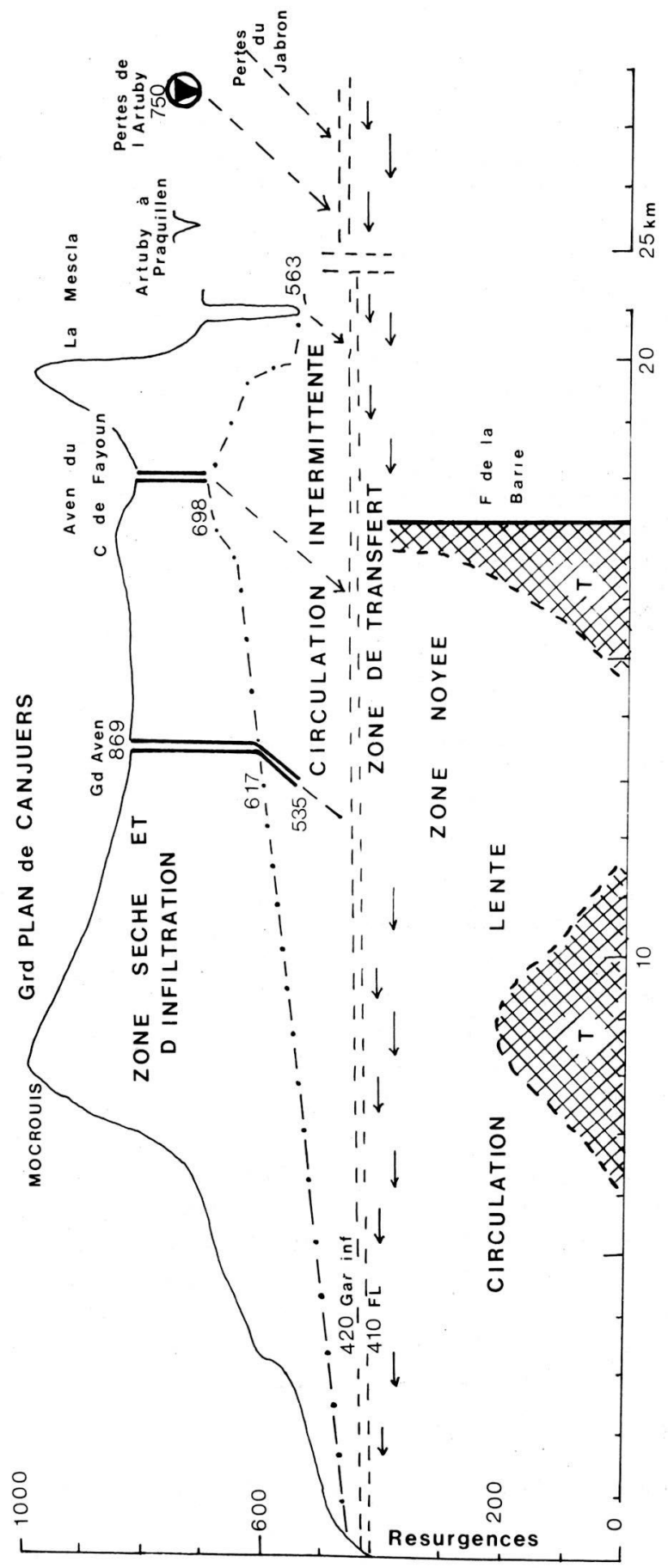


témoigne l'importance du volume dynamique stocké dans le karst (de l'ordre de 70 × $106 \mathrm{~m}^{3}$, Mangin 1975, p. 71). La zone noyée n'envahit nulle part les réseaux accessibles, ni au fond du Grand Aven de Canjuers (535 m), ni dans le Canyon de l'Artuby où ni source, ni perte-émergence («estavelle») n'ont été signalées. L'évolution souterraine s'effectue en fonction du niveau de la résurgence, et non du fond actuel du canyon. Nous avons montré (Nicod 1967) que c'est la surrection des Plans, et l'abaissement conjoint du niveau des exutoires au cours du Quaternaire (attesté par la position des émergences de trop-plein des Garrubys) qui sont responsable de cette disjonction des niveaux de base dont a été victime le cours inférieur de l'Artuby.

3 - Le cas du Verdon est différent. L'examen structural montre que des pertes sont possibles à l'amont du Canyon, et particulièrement à la traversée du champ de faille de la Mescla aux Cavaliers (une d'elle avait eté signalée par E. A. Martel). A l'aval par contre, le canyon se trouve sur le flanc du synclinal de Mayreste; l'anticlinal du Grand Margès l'isole du système karstique des Plans, il n'y a donc pas de possibilité d'absorption; au contraire le canyon sert de drain (3), et le profil d'équilibre subaérien est, à l'exception du passage étroit de l'Imbut, parfaitement réalisé.

\section{CONCIUSION.}

I.a notion de niveau de base karstique majeur est éclairée par l'analyse des conditions d'écoulement dans le fond des canyons.

('e niveau est de caractère hydrodynamique: il est en relation avec le niveau de l'exutoire, et sa perte dépend des pertes de charge; il se situe à la partie supérieure de la zone noyée, dans la zone de transfert horizontal prédominant (ex. zone "vadose»). Il varie suivant les conditions hydrologiques, mais son balancement est limité vers le bas par la position même des exurgences. La zone de transfert est le siège des actions dynamiques, et il parâit utile de rappeler le concept de Mangin selon lequel «le niveau de base d'un système karstique apparâit ainsi comme la limite au delà de laquelle l'énergie potentielle a été entièrement transtorméen (1975, p III, p. 105).

Suivant que le niveau de base karstique majeur coüncide avec le profil subaérien, ou non, lécoulement é les actions érosives et corrosives sont coordonnées dans le fond du canyon ou au contraire le fond du canyon n'évolue qu'en temps de crue, el présente même un profil des plus irréguliers (cas de l'Artuby).

I 'abaisement du niveau des exutoires perennes, ou la surélévation de la masse calcaire, ou cer deus causes reunies (Plans de (anjuers) sont responsables

3 - Des émergences permanentes et temporaires sont signalées à l'aval des Cavaliers, de plus la Mainmorte, qui sort du synclinal crétacé de la Palud, tombe en cascade dans le Canyon. 


\author{
de la disjonction entre profil d'équilibre subaérien et niveau de base souter- \\ rain.
}

\begin{abstract}
RÉSUMÉ
Dans un massif karstique traversé par un canyon dans lequel coule une rivière allogène, il y a plusieurs niveaux de base; surtout si les calcaires se développent largement sous le talweg. La complexité qu'il y a de les caractériser avec précision est unanimement reconnue et se retrouve dans les définitions classiquement proposées. A partir de l'exemple des trois principaux canyons du Languedoc oriental, Ardèche, Cèze et Gard, et de ceux des Plans de Provence, Verdon et Artuby, on constate actuellement que:

a) Lorsqu'un cours d'eau allogène (épigé) est pérenne et sans pertes notables, il y a correspondance globale entre le niveau de base fluviatile s.l. et le niveau de base karstique majeur, constitué approximativement par la zone de transfert horizontal, au sommet de la zone noyée.

b) Lorsqu'un cours d'eau allogène (épigé), ne coule pas par suite de prélèvements karstiques (cours d'eau temporaire ou épisodique), il n'y a pas de correspondance entre ces deux niveaux de base. Le niveau de base fluviatile s.l. est temporairement actif. Quant au niveau de base karstique majeur il se place au niveau des circulations karstiques pérennes sous le talweg sec ou en dehors de lui, commandé à l'aval par la position des sources pérennes majeures drainant le karst.
\end{abstract}

\title{
BIBLIOGRAPHIE
}

BAKALOWICZ M., 1975: Géochimie des eaux karstiques et karstification. Ann. de Spéléo., 30, 4 pp. 581-589;

BLEAHU M.D., 1974: Morfologia carstica. Ed.Stiintificá, Bucarest, 590 p., cf. pp. 305-541.

CASTANY G., 1967: Traité pratique des eaux souterraines. Ed. Dunod, Paris, 661 p., cf. chap 22.

CASTANY G. et MARGAT J., 1974: Dictionnaire français d'hydrogéologie Rapport B.R.G.M.., Orléans, 74 SGN 253 AME.

CAVAILLE A., 1964: Observations sur l'évolution des grottes. Intern. Journal of Speleo. I. 1-2, pp. $71-100$

COULET E., 1975: Morphologie des plaines et garrigues du Languedoc méditerranéen. Thèse' docto. E.Lettres. Inst.Géog. Uni.P.Valéry. Montpellier, 3 vol. 2042 p., 177 fig. 17 phot,. è 1 carte h.t., cf. chap. 4, pp. 287-456.

CVIJIC' J., 1918: Hydrologie souterraine et évolution morphologique du karst. Re'l. Trav.Inst.Géog. Alpine Grenoble 6, 4, 56 p.

CVIJIC' J., 1960: La géographie des terrains calcaires Monog. Ac - Serhe Sci. Arts. Bclgrade, I 341, 212 p. (écrit en 1927).

DEMANGEOT J., 1965: Géomorphologie des Abruzzes adriatiques, Paris, C.N.R.S., 403 p., pp. 187-189.

DUBOIS P., 1969 : Sur les karstifications tertiaire et quaternaire du Bas I anguedoc. Actes s Cong. Inter.de Spéléo., Stuttgart, M 37/1, 7 p., 1 carte.

EK C.M., 1969: Facteurs, processus et morphologie karstique dans les calcaires paleoroiques de la Belgique. Thèse Doct.Sci.Lab.Géolo.Phys. Univ. Liège, $1^{\circ}$ partie: I és eaux souterraines èt superficielles, leur action sur les carbonates $200 \mathrm{p}$.

FABRE G., 1976: Sur l'alimentation des écoulements karstiques dans les canyons d'apres l'interprétation des traçages colorants et géochimiques. Actes du $2^{\circ}$ Coll. d'Hydrol. e'n pays calcuire' Besançon, octobre 1976.

FABRE G., 1977 - Niveaux de base actuels dans les trois principaux canyons du I anguedoc oriental: l'Ardéche, la Cèze et le Gard; Assoc. Géogr. Franc. Paris, 1977, n 445-446, p.237-245.

FENELON P., 1967: Vocabulaire français des phenomines harstiques. Me'm. et des du.C.R.D.C.G., 4, pp. 13-68

FENELON P., 1975: Niveaux de base karstique et geomorphologiques. In "litudes géog.". Me. langes (i.Viers, Univ. Toulouse, TI, pp. 255-261 
GAMS I., 1974: Kras. Izdala Slosvenka matica, V, Ljubljana, 379 p.

GEZE B., 1965: Les conditions hydrogéologiques des roches calcaires. Chron. d'Hydrogeol.B.R.G.M.., n ${ }^{\circ}$, pp. 9-39

GEZE B., 1973: Lexique des termes français de spéléologie physique et de karstologie. Ann.de Spéléol. 28, I, Pp. 1-20

GUERIN R., 1973: Un exemple du rôle de la tectonique et de la micro-tectonique dans la géométrie des écoulements karstiques fossiles et actuels: le Bas Vivarais calcaire. Thèse docto. $3^{\circ}$ Cycle, Inst. Géol.Fac.Sc., Montpellier, 110 p. 132 fi. 2 cartes h.t., annexe 8 p. et 15 fig.

ILIE I.D., 1970: Geomorfologia carstului. Centr. mult. univ.Bucarest, 548 p.

JAKUCS L., 1971: A karasztok morfogenetikaja. Akad. Kiado. Budapest, 310 p.

LLOPIS LLADO N., 1970: Fundamentos de hydrogeologia carstica. Ed. Blume, Madrid 269 p. MANGIN A., 1974 - 1975: Contribution à l'étude hydrodynamique des aquifères karstiques. Thèse doct. E. Sci. Dijon. Ann. de Spéléol. 29, 3, pp. 283-332, 4, pp. 495-601, 30, I pp. $21-124$. NICOD J., 1967: Recherches morphologiques en Basse Provence calcaire. Thèse doct. Lettres Inst.Géog.Fac.Lettres. Aix en Provence, Et. et trav. de Méditerranée, 5 Imp L.jean, Gap, 577 p, 12 phot, et 7 cartes h.t.

NICOD J., 1969: Sur le régime de quelques sources karstiques de Basse-Provence, le problème des réservoirs karstiques; Comité des trav. hist. et schientif., Bull. Sect. Géographie, LXXX 1967, p. 257-320.

PALOC H., et coll. 1975: Glossaire d'hydrogéologie du karst. In «Hydrogeology of karstic terrains», publ.I.A.H., Comm. Karst, Paris, pp. 151-186.

SMITH D., 1971: The concepts of water flow and water tables in limestones. Trans. Cave Res.Group of Great Britain, 13, 2, p. 95-99.

SWEETING M.M., 1972: Karst landforms. Ed. Macmillian, Londres 362 p.

VANDENBERGHE A., 1964: Remarques sur les théories karstiques. Bull. B.R.G.M.., 2, pp. 3350. 